\title{
Temporal Scaling of Optical Rogue Waves in Unidirectional Ring Fiber Laser
}

\author{
Hani Kbashi, Stanislav A. Kolpakov, and Sergey V. Sergeyev* \\ School of Engineering and Applied Science, Aston University, Aston Triangle, Birmingham, B4 7ET, UK \\ *Corresponding author: s.sergeyev@aston.co.uk
}

\begin{abstract}
A fiber mode-lock laser allows generation of the optical rogue wave (ORW) at different time scales. The criteria for distinguishing between them is a comparison of the event lifetime with the main characteristic time of the system. The characteristic time can be estimated from the decay of an autocorrelation function (AF).

Thus, in comparison with AF characteristic time, fast optical rogue wave (FORW) events have duration less than the AF decay time and it appeared due to pulse-pulse interaction and nonlinear pulses dynamics. While slow optical rogue wave (SORW) have a duration much more longer than the decay time of the AF which it papered due to hopping between different attractors. Switching between regimes can be managed by change the artificial birefringence that induced in a laser cavity. For understanding the role playing by the periodical amplification and the resonator, we have performed an unidirectional fiber laser experiments without a saturable absorber. This laser experiment allowed to generate of most of the RW patterns which were either observed experimentally or predicted theoretically. In this way, we have observed the generation of an FORW along with SORW under similar conditions. Most of the patterns were found to be mutually exclusive which means that only one RW mechanism was realized in each regime of generation.
\end{abstract}

Keywords: rogue wave, fiber laser, slow rogue wave, fast rogue wave, rogue wave's interaction, rogue waves collision.

\section{INTRODUCTION}

A rogue wave as a concept has been initially introduced in oceanography to describe rare events, colloquially called "freak" waves. These waves have amplitudes that were much larger than average ones that resulted in destructive impact in nature and society [1]. Investigation of mechanisms which cause appearing of rogue wave (RW) has practical importance due to the damage which they cause in real-world scenarios. Meanwhile, an intrinsic scarcity of events, as well as evident technical difficulties to perform full-scale experiments, are the main obstacles for understand and predict RWs. Mode-locked lasers are perfect candidates to be test-bed systems for investigating conditions for RWs' emergence and mitigation [2]. Lasers with a high repetition rate of pulses provide an opportunity to obtain a huge amount of data under laboratory-controlled conditions in relatively short time. Previously, in the context of mode-locked fiber lasers, the ORW mostly have been observed at regimes of chaotic bunches of noise-like pulses or soliton rain [3]. Also, it has been found that ORW can be generated in mode-locked lasers as a result of the interaction of dissipative solitons interacting through overlapping of their tails or by dispersive pulses [4]. An extensive study of the mechanisms of formation of ORWs have been done either experimentally or theoretically in fiber lasers with nonlinearly driven cavities [5], Raman fiber amplifiers and lasers [6] and fiber lasers via modulation of pump [7]. In the other hand, the hopping has also been investigated as a source of ORWs. The most of the previous experiments were done at high pump power over different experimental condition and using a different cavity design being mostly limited to solitons-soliton interaction.

Unlike in previous publications, we have used the same unidirectional laser experiments to observe both of SORW and FORW at pump powers close to the lasing threshold. The RW's generated was controlled by changing the birefringence in the cavity and the polarization of pump by means of polarization controllers. We have observed two classes of RWs patterns. The first one comprises the RW patterns duration faster than the characteristic time of the system which we have named fast optical rogue waves (FORW) and the second one when the RW events duration significantly longer than the characteristic time (slow optical rogue waves or SORW). Following we have classified and illustrated patterns which we have observed during the experiment. We have arranged the patterns by the frequency of observation, beginning with the most common patterns and ending with the rarest ones.

\section{EXPERIMENTAL SETUP}

The proposed unidirectional fiber ring laser was built as it is illustrated in Fig. 1. Unlike in common modelocking schemes with nonlinear polarization rotation, we have placed only one in-line cavity polarization controller $(\mathrm{CPoC})$ inside the cavity. Another controller $(\mathrm{PPoC})$ was used to control the polarization of pump. The cavity comprises $1 \mathrm{~m}$ of $\mathrm{Er}^{3+}$ doped fiber Liekki Er80-8/125 and $614 \mathrm{~m}$ of single mode fiber SMF-28 $\left(\beta_{2}=-21 \mathrm{ps}^{2} / \mathrm{km}\right)$. The laser cavity was made long to diminish the repetition frequency and exclude the influence 
of coherence. An optical isolator with $51 \mathrm{~dB}$ back losses has been used to assure the unidirectional lasing. The $80 / 20$ fiber coupler for $1550 \mathrm{~nm}$ was used to sample a portion of the signal wave. The pulses that have been generated were not solitons. Additionally, we have tune the pulse length by inducing artificial losses of $\sim 5 \mathrm{~dB}$ bending the fiber in the cavity. These additional manual tuning have allowed us to resolve the pulse-pulse interaction in detail. The laser has a threshold of $16 \mathrm{~mW}$. The angles of the polarization controllers were measured relative to the vertical position.

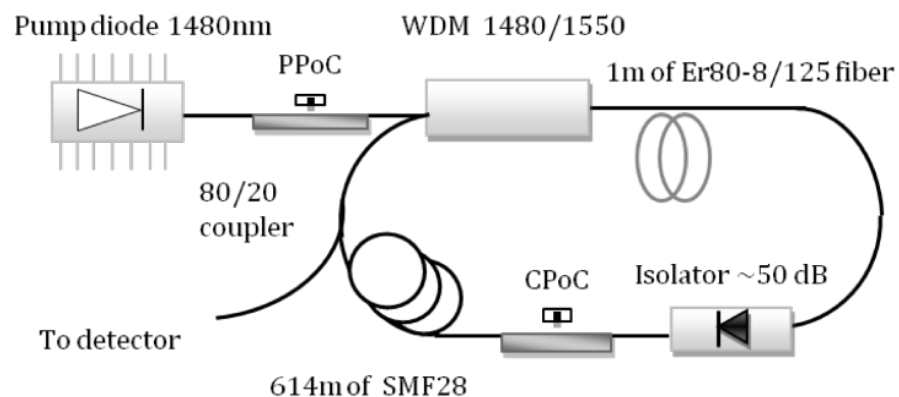

Figure 1. The schematic of the unidirectional fiber laser.

\section{RESULTS AND DISCUSSION}

Autocorrelation functions were taken using oscilloscope trace with the length of 50 million points with a resolution of $0.1 \mathrm{~ns}$. Fast oscillations which correspond with round-trip period and polarization instability have been removed from the autocorrelation function using a peak detection algorithm. For different laser regimes, the filtered AFs have different shapes, for example when the laser generate a "lonely" and "three sisters" FORW patterns, AF has an exponential shape (Fig. 2a), whereas, when the laser generates SORW, the autocorrelation function is linear. The shape of AF provided an information about characteristic times of different processes which have a place in the laser cavity.
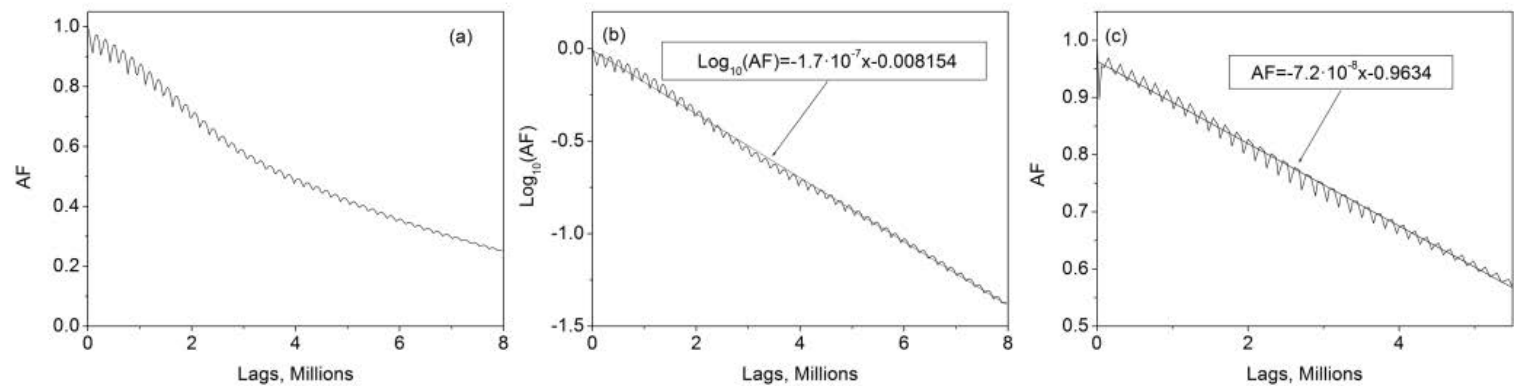

Figure 2. Autocorrelation functions: (a) - AF in the situation when only FORW were observed, (b) - Log of the same function as in (a) and the linear fitting, (c) - the AF in the case when only SORW patterns were observed. In all cases, the length on the one Lag was $0.1 \mathrm{~ns}$.

The small oscillations on the curve of AF correspond with the oscillations of the power with $\mathrm{kHz}$ frequency. The linear fitting $(y=a t+b)$ of the AF gave us the characteristic time $\left(\mathrm{T}_{\mathrm{c}}=1 / a\right.$ in a sec); here, we used $x$ instead of $t$ in insets of Fig. 2 because of the scale of the horizontal axis is in Lags ( $0.1 \mathrm{~ns}$ per Lag). The characteristic times for the case (a) and case (c) were $33 \mathrm{~ms}$ and $9.6 \mathrm{~ms}$ correspondingly (the round trip was $3.0773 \mu \mathrm{s}$ ). These times are similar to the lifetime of the laser level and supplied the information about how long the system "remember" the initially induced perturbation. When the lifetime of the perturbation is significantly longer than the first characteristic time, the system lost the information about how the perturbation was developed and thus the tail of the perturbation is independent on the front of the perturbation. Meanwhile in the opposite case (FORW), the shape of the perturbation is auto-correlated; this situation is similar with the temporal coherence.

The responsible mechanism for emerging of SORW patterns is illustrated in Fig. 3. The system seemingly has four amplitude attractors; therefore, the hopping between them (with appropriate frequency and level of background noise) can lead to surpassing of RW threshold. This situation apparently was similar to the situation that observed during pump modulation [8]. Probably, this happens because of the intrinsic low-frequency modulation of a signal power (see Fig. 2) performed seemingly to an external pump modulation. In the case when FORWs were generated, the patterns have affected by periodic amplification in each round-trip, which can be considered as a kind of "memory". During the second scenario (SORW), the system "forget" how a pattern has begun meanwhile the pattern is still active. This strongly affects the statistics of the RWs and the FORW scenario excludes the SORW one. 


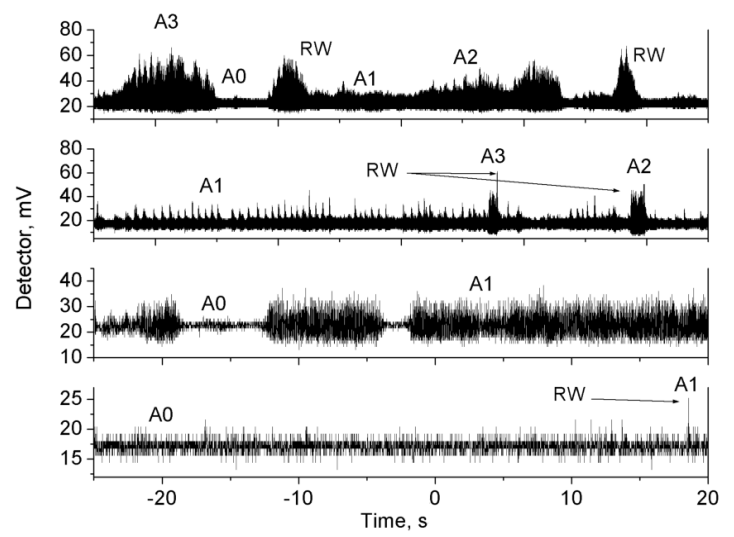

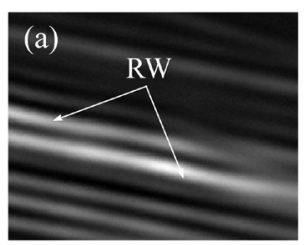

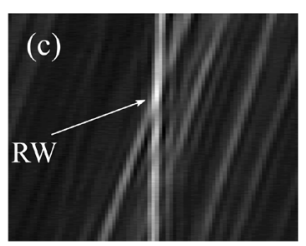

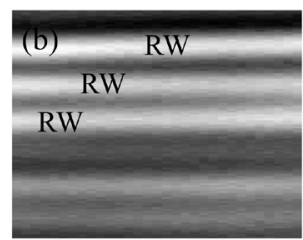

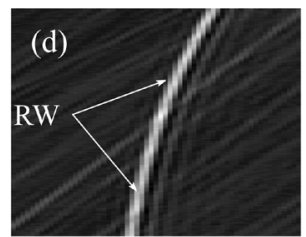

Figure 3. On left the intermittencies at a slow temporal scale. The system apparently has four attractors labeled with A0 -A1. At right four fast patterns a - "twins"; b - "three sisters"; c - "cross" pattern and $d$ - "accelerated $R W^{\prime \prime}$.

In the case of a "cross" pattern (see the right inset in Fig. 3c) the relative speed of the approaching pulses is high $(>10 \mathrm{~m} / \mathrm{s})$ that the pulses have no time to interact (typically $\sim 100$ round-trips is needed). Therefore, the "cross" pattern can be considered as a limit case of the same pulse-pulse interaction mechanism. The opposite limit case constitutes "twins" patterns (Fig. 3a) when the relative pulse speed is close to zero and two pulse interact during thousands of round trips while form two parallel oscillating FORWs. Moreover, unlike the "cross" pattern which was produced by the direct collision between two pulses when the pulses have no time to interact, we have observed that the "accelerating wave" pattern (Fig. 3d) appeared because of resonance interaction between three pulses.
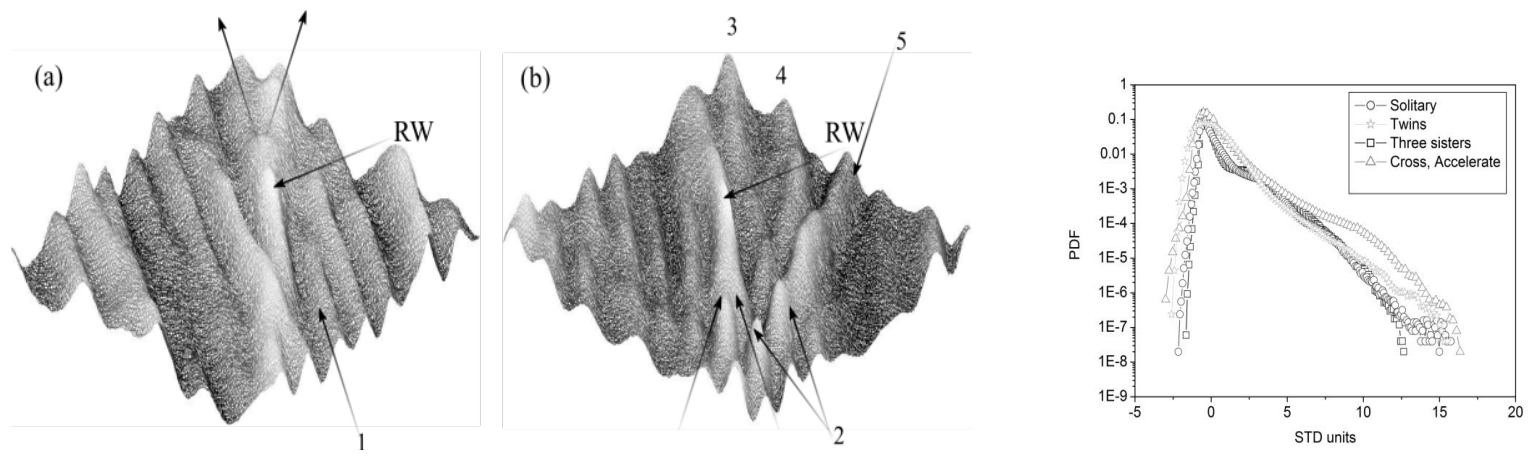

Figure 4. Fast "solitary" RW pattern: (a) the RW pattern splits in two SWH after interacting with the pulse "1" and (b) the RW pattern which appears as a result of a resonance merging of two pulses. The "spike" SWH pattern "2" appears as a result of a collision between pulses "3"," 4 " and "5". On the right insert are PDFs for FORW patterns.

The mechanisms which are responsible for the emergence of the FORW patterns (Fig. 4a and Fig. 4b) were based on resonance pulse-pulse interaction when two slowly approaching pulses exchanged their energies. This mechanism illustrated in Fig. 4a is elucidate the splitting of the RW pattern in two significant wave height (SWH) pulses, meanwhile Fig. $4 \mathrm{~b}$ shows the creation of the "solitary" RW pattern from two merging SWH pulses. In the case of a "solitary" or "lonely" RW pattern which is known mechanism of interaction between pulses that propagate at different speeds. The similar mechanisms were observed for "twins", "three sisters" and "accelerated" patterns (compare with the right inset in Fig. 3). "lonely" and "three sisters" patterns have different probability distribution function (PDF) from the shape of the PDF of the "twins" patterns as shown in Fig. 4c. and Table 1.

Finally, we have classified the ORW patterns as it is shown in Fig. 5 and in Table 1. The Venn diagram in Fig. 5b which illustrates the possible logic relations between different patterns. For example, the "lonely" and "three sisters" patterns were observed at the same lasing regime, whereas, the "twins" patterns have emerged in different regimes particularly when neither "lonely" nor "three sisters" were observed. We have classified "twins" pattern as an FORW instead it long lifetime which was very close to $T_{\mathrm{c}}\left(T_{\mathrm{c}} \sim 30 \mathrm{~ms}\right.$ and typical pattern duration time $\left.T_{\mathrm{p}} \sim 5 \mathrm{~ms}\right)$. 
(a)

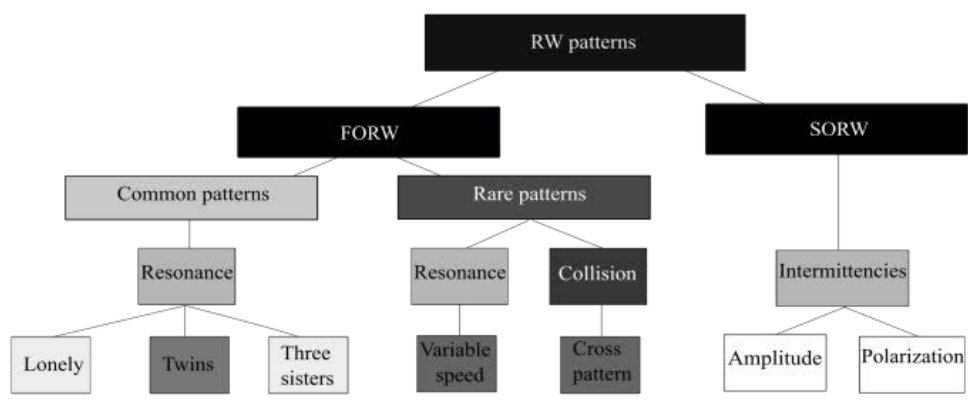

(b)

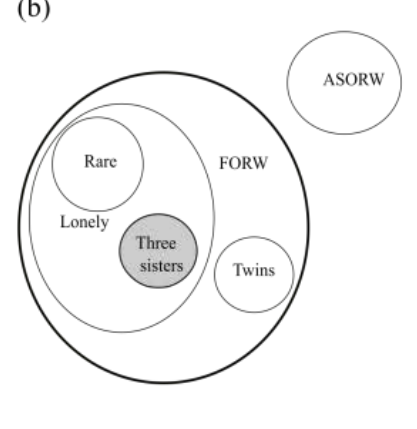

Figure 5. Classification of $R W$ patterns: (a) - the classification of the patterns (the polarization $R W$ were detected in a laser with saturable absorber) and (b) - A Venn diagram.

Table 1. Classification of $R W$ patterns.

\begin{tabular}{|c|c|c|c|}
\hline Pattern & Lifetime (round-trips) & Likelihood $\left(\mathrm{N}_{\mathrm{e}} / \mathrm{N}_{\mathrm{p}}\right)$ & Mechanism \\
\hline Lonely & Hundreds & $\sim 0.6$ & Pulse-pulse interaction \\
\hline Twins & Thousands & $\sim 0.3$ & Pulse-pulse interaction \\
\hline Three sisters & Hundreds & $\sim 0.1$ & Pulse-pulse interaction \\
\hline Accelerated $^{*}$ & Hundreds & $<0.01$ & Pulse-pulse interaction \\
\hline Cross ${ }^{*}$ & $<10^{-4}$ & $<0.01$ & Pulse-pulse collision \\
\hline PSORW $^{* * *}[9]$ & Hundreds of thousands & $\mathrm{NA}^{* *}$ & Polarization trapping \\
\hline ASORW & Millions & $\mathrm{NA}^{* *}$ & Hopping \\
\hline
\end{tabular}

*Pattern was detected only for regime similar to soliton rain.

${ }^{* *}$ Not enough statistics, likelihood is not available.

${ }^{* * *}$ Pattern was observed in a laser with a saturable absorber.

\section{CONCLUSIONS}

In conclusion, two groups of RW patterns have been observed in unidirectional self-mode-locked fiber laser due to polarization dynamics in the cavity. The first group of the pattern (FORW) is composed of pulses with the duration significantly less than the AF characteristic time and it appeared due to pulse-pulse interaction and nonlinear pulses dynamics. Meanwhile, the second one (SORW) consist of the oscillations with the duration significantly longer than the characteristic time. The mechanism SORW patterns are different from the mechanism of the FORW patterns and can be explained in terms of hopping between different attractors in multi-state oscillation system. Most of the patterns were found to be mutually exclusive which means that only one RW mechanism was realized each time. This, in turn, means that all patterns of pulse-pulse interaction ("lonely", "twins", three sisters" and "cross") probably are different cases of the same interaction mechanism.

Furthermore, the FORW patterns have been classified into three different subgroups. The "lonely" and "three sisters" patterns that have the same shape of PDF and trend to appear at the same positions of polarization controllers although the "three sisters" pattern has significantly smaller likelihood than the "lonely" one. The "twins" pattern needs a specific polarization regime and has not been observed along with the "lonely" or "three sisters" patterns. The "rare" patterns appear only in one lasing regime which is similar to a "soliton rain" regime and can be observed along with "lonely" patterns.

\section{REFERENCES}

[1] C. Kharif, E. Pelinovsky, and A. Slunyaev: Rogue Waves in the Ocean, Springer, Heidelberg, 2009.

[2] C. Lecaplain, Ph. Grelu, J. M. Soto-Crespo, and N. Akhmediev: Dissipative rogue waves generated by chaotic pulse bunching in a mode-locked laser, Physical Review Letters 108, 233901,2012.

[3] S. Chouli, and Ph. Grelu: Rains of solitons in a fiber laser, Optics Express, vol. 17, no. 14, 2009.

[4] C. Lecaplain, Ph. Grelu, J. M. Soto-Crespo, and N. Akhmediev: Dissipative rogue wave generation in multiple-pulsing mode-locked fiber laser, Journal of Optics, vol. 15, 064005, 2013.

[5] D. R. Solli, C. Ropers, P. Koonath, and B. Jalali: Optical rogue waves, Nature 450, 1054, 2007.

[6] K. Hammani, C. Finot, J. M. Dudley, and G. Millot: Optical rogue-wave-like extreme value fluctuations in fiber Raman amplifiers, Optics Express 16, (21), 16467-16474, 2008.

[7] A. N. Pisarchik, R. Jaimes-Reategui, R. Sevilla-Escoboza, G. Huerta-Cuellar, and M. Taki: Rogue waves in a multistable system, Physical Review Letters 107, (27), 274101, 2011.

[8] G. Huerta-Cuellar, A. N. Pisarchik, and Y. O. Barmenkov: Experimental characterization of hopping dynamics in a multistable fiber laser, Physical Review E. 78, 035202(R), 2008.

[9] S. Sergeyev, S. Kolpakov, Ch. Mou, G. Jacobsen, S. Popov, and V. Kalashnikov: Slow deterministic vector rogue waves, SPIE Proceedings, vol. 9732, 2017. 